\title{
CD64 Index as a Predictor of Outcome for Children with Systemic Inflammatory Response Syndrome (SIRS)
}

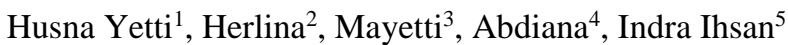 \\ \{husnayetti@med.unand.ac.id ${ }^{1}$, herlina2000@ymail.com², mayettispa@yahoo.com³ \\ abdiana.fk.unand@gmail.com ${ }^{4}$, indraihsan@yahoo.co.id ${ }^{5}$ \} \\ Department of Public Health and Community Medicine, Faculty of Medicine, Andalas University, \\ Padang, Indonesia ${ }^{1,4}$ \\ Department of Pediatrics, Faculty of Medicine ${ }^{2,3}$ \\ Andalas University/ M. Djamil General Hospital, Padang, Indonesia ${ }^{5}$
}

\begin{abstract}
Sepsis is one of the major causes of mortality and morbidity in children. The study aimed to assess the prognostic value and difference of Neutrophil CD64 index regarding sepsis outcome. Methods of this study were a prospective observational study in 62 children with Systemic Inflammatory Response Syndrome (SIRS) were conducted in ER and Pediatric Ward Dr. M. Djamil Hospital, Padang. We collected blood samples to measure Neutrophil CD64 index and blood cultures were drawn to confirm infection. Patients were observed clinically until discharge from the hospital. Based on the clinical course and laboratory test results, patients were categorized as having unproven sepsis, sepsis, septic shock, and nonsurvivor. The average Neutrophil CD64 index were $1.13 \pm 0.32 ; 1.71 \pm 0.23 ; 2.29 \pm 0.71 ; 2.86 \pm 1.10$ for unproven sepsis, sepsis, septic shock, and nonsurvivors, respectively. The area under the curve (AUC) for hospital mortality was 0.863 . At a cut-off level of 1.705 sensitivity was $71.4 \%$ and specificity was $72.9 \%$. Patients with CD64 index level higher than 1.705 had 6.7-fold higher probability of dying. The odd ratio is 6.7 (95\% CI 1.79-25.27). Neutrophil CD64 was significantly elevated as a severe outcome of sepsis. This result indicates that neutrophil CD64 may be used as a biomarker for predicting mortality.
\end{abstract}

Keywords: CD64 neutrophil, SIRS, Sepsis, Pediatric, Prognosis.

\section{Introduction}

Sepsis is the prominent causal of morbidity and mortality in children worldwide including developing countries [1]. The prevalence and mortality rate were varied across countries [2]. Sepsis is defined as life-threatening organ dysfunction or systemic inflammatory response syndrome (SIRS) caused by dysregulated host response to infection [3,4]. Its symptoms are similar to SIRS without infection [5]. The disease could progress to severe sepsis or septic shock and mortality.

Previous studies have demonstrated that some biomarker could predict the severity and/or sepsis outcome such as Procalcitonin, TNF, LPS, IL-6. IL-8, CD64 [6-8]. However, in some studies evaluating the prognostic value of these markers, found that IL-6 [8]; PCT and CRP [9] were not the ideal predictor of prognosis. 
One of a biomarker that is widely assessed is CD64. CD64 (FcyR1) is a membrane glycoprotein, which is expressed mainly on monocytes and macrophages with low concentration on the surface of non-activated neutrophils. Neutrophil CD64 (nCD64) expression can be distinctly elevated at the onset of sepsis [10], and assessment of this protein expression is superior to standard laboratory tests (neutrophilia, band forms, etc.) [11,12] for early detection of sepsis.

A study by Groselj-Grenj et al. (2008) also shown that expression of CD64 on neutrophil is elevated in patients with sepsis compared with noninfectious SIRS [13]. Hsu et al. 2011 observed an association of CD64 to mortality [14]. Nevertheless, the prognostic value of CD64 in children with SIRS is still uncertain, so the study aimed to know the role of CD64 Neutrophil index to predict sepsis outcome in children with SIRS.

\section{Materials and Methods}

\subsection{Subject}

A prospective observational study conducted throughout 5 months from March to July 2017. We included child patients (age 1-14 years) admitted to the Emergency Room (ER) of Dr. M. Djamil Hospital affiliated with the Faculty of Medicine, Andalas University, who presented with features of SIRS. Exclusion criteria were children with malignancy (lymphoma, carcinoma, sarcoma, leukemia), HIV/AIDS, autoimmune disease (juvenile chronic arthritis, ankylosing spondylitis, psoriatic arthritis, systemic vasculitis, polymyalgia rheumatic, Reiter disease, Crohn disease). Informed consent was obtained from the parents before enrollment in the study. Sixty-two consecutive patients with SIRS and clinically suspected infections were eligible for enrollment. SIRS was defined according to the international pediatric sepsis consensus conference definitions [4]. Sepsis and septic shock were defined according to the Third International Consensus Definitions for Sepsis and Septic Shock 2016 (Sepsis-3) [3]. The negative result of blood culture was considered as unproven sepsis. Patients were monitored during their hospitalization at the pediatric ward. Clinical outcomes were assessed based on two parameters survival and nonsurvival. Survival patients had improvement condition and were hemodynamically stable at the time of discharge. The institutes' ethical committee approved this study.

\subsection{Assessment of neutrophil CD64 index and blood culture}

Patients` blood samples were taken on the first-day admission to the ER. Neutrophil CD64 index was analyzed by flow cytometry performed on a FacsScanto ${ }^{\mathrm{TM}}$ flow cytometer (Becton Dickinson, NY, USA) with $100 \mu$ l EDTA-anticoagulated whole blood. CD64-APC (clone 10.1.1) antibodies from Miltenyi Biotec were used according to the manufacturer's instructions. Blood culture was performed in medium BacT/ALERT using automatic machine BacT/ALERT 3D Microbial Identification System.

\subsection{Statistical analysis}

Continuous variables are presented as mean values \pm SD or medians and ranges, and categorical variables are expressed as a percentage. Normality of continuous variables was tested using 
Kolmogorov-Smirnov test. We compared the variables between the two groups using the independent $t$-test or Mann-Whitney U-test for continuous variables and a X test or the Fisher's exact test for categorical variables. Receiver operating characteristic (ROC) curves were used to examine the performance of variables in predicting hospital mortality. The area under the curve (AUC) was calculated from the ROC curve. SPSS version 21 (IBM, Chicago, USA) was used for statistical analysis. $\mathrm{P}<0.05$ was considered to be statistically significant.

\section{Results}

The study showed that from the SIRS patients, there were 48 patients $(77.4 \%)$ were survived and 14 patients $(22.6 \%)$ were non-survived. Even though sex was not statistically different between boys and girls in regarded to sepsis outcome, girls (29\%) who non-survived were more common than boys $(16.1 \%)$ (Table 1$)$. Statistically, there were no differences between age group patients in regard to sepsis outcome $(\mathrm{p}=0.734)$. Patients who age $>1$ month to 1 year $(27,3 \%)$ were more common in non-survived compared to age $2-5$ years $(23,1 \%)$ and age 6-12 years $(14,3 \%)$.

Table 1. Demographic and Clinical Characteristics of Patients with Sepsis According to Outcome.

\begin{tabular}{|c|c|c|c|c|}
\hline Variable & $\begin{array}{l}\text { All patients } \\
(\mathrm{n}=62)\end{array}$ & $\begin{array}{l}\text { Survivors } \\
(\mathrm{n}=\%)\end{array}$ & $\begin{array}{l}\text { Nonsurvivors } \\
(\mathrm{n}=\%)\end{array}$ & $P$ value \\
\hline \multicolumn{5}{|l|}{$\operatorname{Sex}, \mathbf{n}(\%)$} \\
\hline Boys & $31(50 \%)$ & $26(83,9 \%)$ & $5(16,1 \%)$ & 0,362 \\
\hline Girls & $31(50 \%)$ & $22(71,0 \%)$ & $9(29,0 \%)$ & \\
\hline \multicolumn{5}{|l|}{ Age } \\
\hline 1 month -1 year & $22(35.5 \%)$ & $16(72,7 \%)$ & $6(27,3 \%)$ & 0,660 \\
\hline $2-5$ years & $26(41.9 \%)$ & $20(76,9 \%)$ & $6(23,1 \%)$ & \\
\hline $6-12$ years & $14(22.6 \%)$ & $12(85,7 \%)$ & $2(14,3 \%)$ & \\
\hline $\begin{array}{l}\text { Long of hospital stay, days, } \\
\text { median (min-max) }\end{array}$ & $7(1-18)$ & $8(5-18)$ & $2(1-17)$ & 0,000 \\
\hline \multicolumn{5}{|l|}{ Disease grade } \\
\hline Unproven sepsis & $38(61.3 \%)$ & $31(81.58 \%)$ & $7(18.42 \%)$ & 0,000 \\
\hline Sepsis & $6(9.68 \%)$ & $6(100 \%)$ & $0(0 \%)$ & \\
\hline Septic shock & $18(29.03 \%)$ & $11(61.11 \%)$ & $7(38.9 \%)$ & \\
\hline \multicolumn{5}{|l|}{ Laboratory results } \\
\hline Hemoglobin $\mathrm{g} / \mathrm{L}$, mean (SD) & $10.55 \pm 1.71$ & $10,8 \pm 1,56$ & $9,68 \pm 1,95$ & 0,029 \\
\hline $\mathrm{WBC} \times 10^{9} / \mathrm{L}$, mean $(\mathrm{SD})$ & $14.978 \pm 8217$ & $13.390 \pm 7061$ & $20.422 \pm 9763$ & 0,004 \\
\hline CD64 index, mean (SD) & $1.78 \pm 0.96$ & $1.47 \pm 0.64$ & $2.86 \pm 1.1$ & 0,000 \\
\hline
\end{tabular}

The length of hospital stay in our study was varied between one to 18 days. In survival SIRS patient, the median length of stay was eight, while two in non-survival SIRS patient, and statistically different between survival and nonsurvival $(\mathrm{p}<0.001)$. In this study, SIRS without sepsis or unproven sepsis $(61.3 \%)$ were more common than sepsis $(9.68 \%)$ and septic shock (29.03\%). The nonsurvivors consisted of 7 patients with negative blood culture and 7 patients with positive blood culture which was considered as a septic shock.

Hemoglobin level in nonsurvivors $(10,8 \pm 1,56 \mathrm{~g} / \mathrm{L})$ were significantly lower compared with survivors $(9,68 \pm 1,95 \mathrm{~g} / \mathrm{L} ; \mathrm{p}=0.029)$ and White blood cell (WBC) count were higher $(20.422 \pm 9763 / \mathrm{L})$ in nonsurvivors compared with survivor patients $(13.390 \pm 7061 / \mathrm{L} ; \mathrm{p}=$ 
0.004). The CD64 index in nonsurvivors $(2.86 \pm 1.1)$ were higher compared with survivors $(1.467 \pm 0.64 ; \mathrm{p}<0.001)$. Receiver operator curves were generated to determine cut-off values for optimal sensitivity and specificity for CD64 index level for the outcome (Fig. 1). The area under the curve (AUC) for the outcome was 0.863; $\mathrm{p}<0.05$, shown that CD64 index is a good predictor of outcome. At a cut-off level of 1.705 , sensitivity was $71.4 \%$, and specificity was $72.9 \%$ (Table 2). Positive and negative predictive values were $72.92 \%$ and $71.43 \%$, respectively. Patient with CD64 index higher than 1.705 had 6.7 -fold higher probability of death than those with lower values. The odd ratio is $6.7 ; 95 \%$ CI 1.79-25.27.

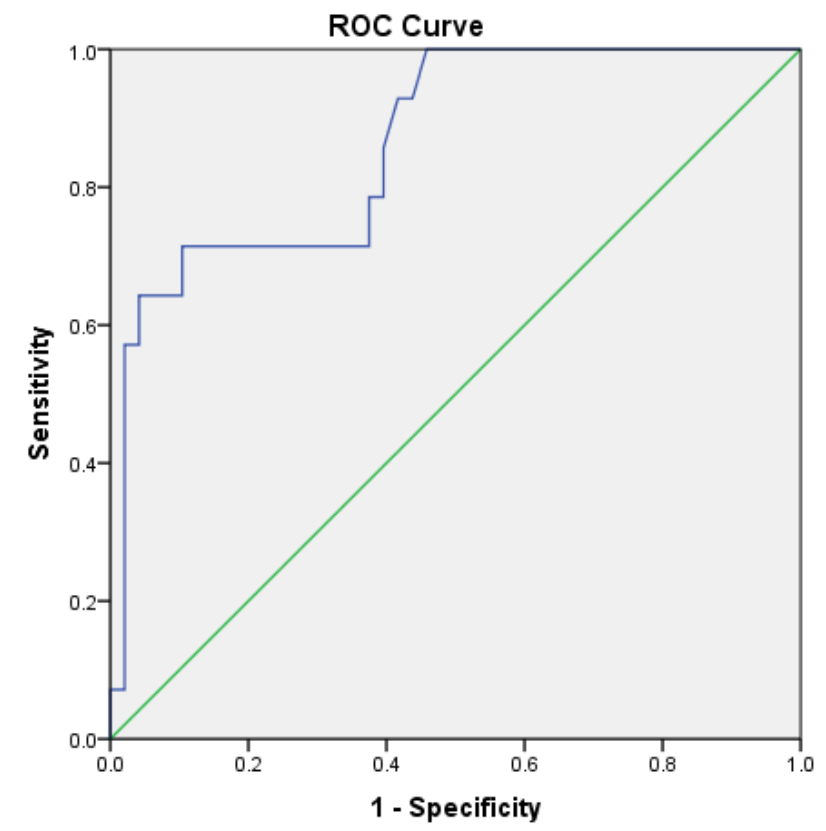

Fig. 1. The accuracy of the neutrophil CD64 index for predicting mortality.

Table 2. Performance of CD64 Index in Predicting Mortality.

\begin{tabular}{cccccccc}
\hline Predictor & AUC & $P$ value & $\begin{array}{c}\text { Cut-off } \\
\text { point }\end{array}$ & OR & $95 \%$ CI & $\begin{array}{c}\text { Sensitivity } \\
(\%)\end{array}$ & $\begin{array}{c}\text { Specificity } \\
(\%)\end{array}$ \\
\hline CD64 index & 0.863 & 0.005 & 1.705 & 6.7 & $1.79-25.27$ & $71.4 \%$ & $72.9 \%$ \\
\hline
\end{tabular}

Furthermore, to compare CD64 index between unproven sepsis, sepsis, septic shock, and nonsurvival, we analyzed the mean difference between each group (Fig. 1). CD64 index increased from $1.13 \pm 0.32$ in SIRS without sepsis or unproven sepsis, to $1.71 \pm 0.23,2.29 \pm 0.71$, and $2.86 \pm 1.10$ in sepsis, septic shock, and nonsurvival patients, respectively. Significant differences were found between unproven sepsis and all other group (sepsis, septic shock and nonsurvival) $(\mathrm{p}<0.001)$, sepsis and nonsurvival $(\mathrm{p}=0.022)$, but not between sepsis and septic shock $(p=0.075)$, and between septic shock and nonsurvival ( $p=0.147)$ (Fig. 2). 


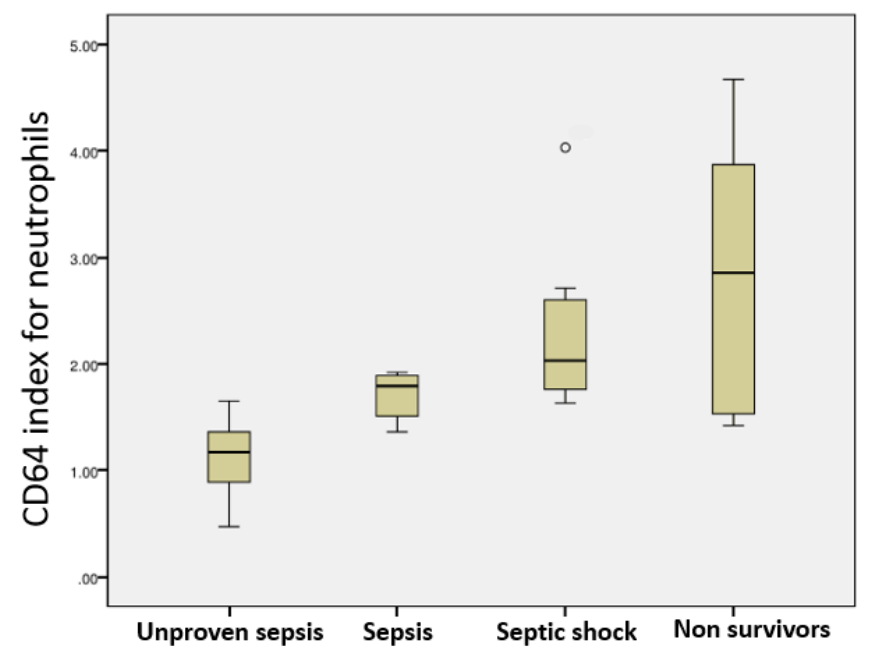

Fig. 2. CD64 index for neutrophil in a different stage of SIRS.

\section{Discussion}

In our study, mortality caused by SIRS was $22.6 \%$, in line with epidemiology study from 26 countries that reported the hospital mortality rate was $25 \%$ and was not differ by age or between developed and resource-limited countries [2]. Regarding the gender, our study was in agreement with Carvalho et al. (2005) [15] and Pavare et al. (2009) [16] which found no gender difference among SIRS patients. Most of the SIRS patients were in the 2-5 year age group $(41.9 \%)$ which is comparable to another study [16].

The median length of stay of survivors was eight days, while two days in nonsurvivors. It indicated that severe condition had a greater probability of mortality [17]. The study by Pawar (2016) demonstrated that significant relationship between mortality and severity of sepsis. However, contrary to our study that found mortality in septic shock patient was comparable with unproven sepsis, i.e. half consisted of septic shock and half unproven sepsis. The study by Pua et al., (2013) corroborated our study that shown culture positivity per se is not independently associated with mortality on multivariate analysis [18]. Delayed in referral patient to the hospital may also contribute to the bad outcome. In our area which is facilities and resources are limited may count for this situation as reported by a previous study [1].

The mean CD64 index in this study was increased as the degree of sepsis deteriorated. Index of CD64 neutrophil was highest in nonsurvival patient and lowest in unproven sepsis. In concordance with a study by Livadity et al. (2006) revealed that CD64 was significantly and positively associated with stages of sepsis [7]. Their study also shown that neutrophil CD64 determined early in the sepsis process, i.e., within the first 24-hours, are superior to other biomarkers in detecting sepsis severity and mortality. Furthermore, the mean index of CD64 in SIRS without sepsis or unproven sepsis were significantly different with other disease state (sepsis, septic shock and nonsurvival). Our study corroborated by the previous study that demonstrated CD64 indexes for neutrophils can discriminated SIRS with sepsis and without sepsis [13]. Additionally, in line with the study by Kobold et al. (2002) that presenting a trend 
towards higher values of neutrophil-CD64 in non-survivors compared with survivors [19]. However, the mean difference of CD64 index between sepsis and septic shock or nonsurvivors; and between septic shock and nonsurvivorsl were not significantly different. This could be explained by an outlier value (4.03) of CD64 index found in one septic shock patient which may be affected by the mean value on this group (Fig.1). This finding were similar with a study by Velasques el al (2013), which showed that although there was a significant correlation between 28 days mortality and CD64 neutrophil in univariat analysis, but in multivariate analysis, there were no significant different [20]. Godnic et al. (2005) also showed a very small difference between values of sepsis and septic shock, so there was no statistical significance but they could distinguish between SIRS and septic shock $(\mathrm{p}=0.038)$ [11]. Contrary to the study by Gosh et al (2018) and Kobold et al. (2002), they demonstrated no significance difference of CD64 between survivor and nonsurvivors. Both of this studies afflicted by the limitation of a small sample size [19,21].

Previous several studies had demonstrated the role of CD64 neutrophil as a predictor of sepsis outcome with variable results. Djorjevic (2015) shown that the index of CD64 neutrophil on the first day was a good outcome predictor [6]. Then, De Jong (2016) demonstrated that CD64 neutrophil could discriminate between a critically ill patient with positive blood culture and negative blood culture and also were correlated with the degree of sepsis [22]. Muzlovic et al. (2016) demonstrated that index CD64 neutrophil could diagnose sepsis and predict 30-day survival in subjects after ventilator-associated pneumonia (VAP) [23]. Meanwhile, Danikas et al. (2008) shown increased expression of CD64 neutrophil in survival sepsis patients compared with nonsurvivors but not correlated with disease outcome [24]. A study by Cid, et al. (2011) also showed higher nCD64 expression in survivors compared to nonsurvivors [25].

Despite the relatively small number of the patients included in this study, we performed ROC curve which showed high sensitivity and specificity, positive and negative predictive value of the CD64 neutrophil index for predicting the prognosis. This in line with previous study by Livaditi (2016), showed AUC 0.75, sensitivity 66.7\%, specificity $73.9 \%$, PPV $72.7 \%$ and NPV $68 \%$ [7]. Study by Chen et al. (2014) demonstrated AUC of 0.752 ( $\mathrm{p}=<0.001$ ), with cut-off value 1.835 , sensitivity $60 \%$, and specificity $80.23 \%$ [26]. Two other studies also reported similar AUC, 0.707 [14] and 0.71 [25]. Relatively different cut-off values were reported in a different study, probably due to different flow cytometry measurement methods [5].

Bias on diagnosis may be a limitation of this study. It may occur because, despite the sepsis guideline, diagnosis depends on clinician`s ability to identify infection and sepsis severity [11]. In agreement with the previous studies which also classified disease by clinical suspicion, as bacterial infection can not be proven due to various reason such as previous use of antibiotics, delayed culture collection and faulty collection technique [27].

\section{Conclusion}

There was a difference of mean CD64 index in children with SIRS on first-day admission in hospital depend on clinical disease state and outcome. The mean of CD64 index was higher in the severe outcome. Thus neutrophil CD64 index could be a prognostic biomarker for children with SIRS.

Conflict of interest. The authors who have taken part in this study declared that they do not have anything to disclose regarding funding or conflict of interest concerning this manuscript. 
Acknowledgments. A grant supported this work (No. 106/BBPT/PNP/FK-Unand-2017) from Ministry of Research, Technology and Higher Education, Republic of Indonesia (non-tax revenue of Medical Faculty, Andalas University).

\section{References}

[1] Kissoon N, Carapetis J.: Pediatric sepsis in the developing world. J Infect. 71 Suppl 1:S21-6. (2015)

[2] Weiss SL, Fitzgerald JC, Pappachan J, et al.: Global epidemiology of pediatric severe sepsis: the sepsis prevalence, outcomes, and therapies study. Am J Respir Crit Care Med. 191(10):1147-57 (2015)

[3] Singer M, Deutschman CS, Seymour CW, Shankar-Hari M, Annane D, Bauer M, et al.: The Third International Consensus Definitions for Sepsis and Septic Shock (Sepsis-3). JAMA. 315(8):801-810 (2016)

[4] Goldstein B, Giroir B, Randolph A.: The members of the International Consensus Conference on Pediatric Sepsis: International Pediatric Sepsis Consensus Conference: Definitions for sepsis and organ dysfunction in pediatrics. Pediatr Crit Care Med, 6(Suppl 3):2-8 (2005)

[5] Hassuna NA, Omar Mousa SM, Hassan EE, Elgezawy E.: The Diagnostic Value of Neutrophil CD64 in Detection of Sepsis in Children. Egyptian Journal of Medical Microbiology. 25(3):25$29(2016)$

[6] Djordjevic D, Pejovic J, Surbatovic M, Jevdjic J, Radakovic S, Veljovic M, et al.: Prognostic value and daily trend of Interleukin-6, neutrophil CD64 expression, C-reactive protein and lipopolysaccharide-binding protein in critically ill patients: reliable predictors of outcome or not? J Med Biochem 34:431-439 (2015)

[7] Livaditi O, Kotanidou A, Psarra A, Dimopoulou I, Sotiropoulou C, Augustatou K, et al.: Neutrophil CD64 expression and serum IL-8: Sensitive early markers of severity and outcome in sepsis. Cytokine 36:283-290 (2006)

[8] Oda S, Hirasawa H, Shiga H, Nakanishi K, Matsuda K, Nakamua M.: Sequential measurement of IL-6 blood levels in patients with systemic inflammatory response syndrome (SIRS/sepsis). Cytokine 29:169-75 (2005)

[9] Clec'h C, Ferriere F, Karoubi P, Fosse JP, Cupa M, Hoang P, et al.: Diagnostic and prognostic value of procalcitonin in patients with septic shock. Crit Care Med. 32(5):1166-9 (2004)

[10] Davis BH. Improved diagnostic approaches to infection/sepsis detection. Expert Rev Mol Diagn. 5(2):193-207 (2005)

[11] Godnic M, Stubjar D, Skvarc M, Jukic T.: Diagnostic and prognostic value of sCD14-STpresepsin for patients admitted to hospital intensive care unit (ICU). Wien Klin Wochenschr doi. 10.1007/s00508-015-0719-5. (2015)

[12] Davis B.: Quantitative neutrophil CD64 expression: promising diagnostic indicator of infection or systemic acute inflammatory response. J Clin Invest Clin Immunol Newslett. 16:121-30 (1996)

[13] Groselj-Grenc M, Ihan A, Derganc M.: Neutrophil and monocyte CD64 and CD163 expression in critically ill neonates and children with sepsis: comparison of fluorescence intensities and calculated indexes. Mediators of Inflammation. Vol 2008, Article ID 202646 (2008)

[14] Hsu KH, Chan MC, Wang JM, Lin LY, Wu CL.: Comparison of fcy receptor expression on neutrophils with procalcitonin for the diagnosis of sepsis in critically ill patients. Respirology. 16:152-60 (2011).

[15] Carvalho PRA, Feldens L, Seitz EE, Rocha T, Soledade M, Trotta A.: Prevalence of systemic inflammatory syndromes at a tertiary pediatric intensive care unit. J Pediatr. 81:143-148 (2005)

[16] Pavare J, Grope I, Gardovska D.: Prevalence of systemic inflammatory response syndrome (SIRS) in hospitalized children: a point prevalence study. BMC Pediatrics. 9:25 (2009)

[17] Pawar A, Raut A, Kalrao V, Jacob J, Godha I, Thomas R.: Etiology and clinical outcomes of neonatal and pediatric sepsis. Arch Pediatr Infect Dis. 4(2):e33602 (2016) 
[18] Phua J, Ngerng WJ, See KC, Tay CK, Kiong T, Lim HF, et al.: Characteristics and outcomes of culture-negative versus culture-positive severe sepsis. Critical Care 2013, 17:R202

[19] Muller Kobold AC, Tulleken JE, Zijlstra JG, Sluiter W, Hermans J, Kallenberg CG, et al.: Leukocyte activation in sepsis; correlations with Neutrophil CD64 disease state and mortality. Intensive Care Med; 6(7):883-92 (2002)

[20] Velasquez S, Matute JD, Gamez LY, Enriquez LE, Gomez ID, Toro F, et al.: Characterization of nCD64 expression in neutrophils and levels of s-TREM-1 and HMGB-1 in patients with suspected infection admitted in an emergency department. Biomedica ;33:643-52 (2013)

[21] Ghosh PS, Singh H, Azim A, Agarwal V, Chaturvedi S, Saran S, et al.: Correlation of neutrophil CD64 with clinical profile and outcome of sepsis patients during intensive care unit stay. Indian J Crit Care Med;22:569-74 (2018)

[22] De Jong E, De Lange DW, Beishuizen A, Van De Ven PM, Girbes ARJ, Huisman A.: Neutrophil CD64 expression as a longitudinal biomarker for severe disease and acute infection in critically ill patients. Int Jnl Lab Hem. 1:1-9 (2016)

[23] Muzlovic I, Ihan A, Stubljar D.: CD64 index on neutrophils can diagnose sepsis and predict 30day survival in subjects after ventilator-associated pneumonia. J Infect Dev Ctries. 10:260-8 (2016)

[24] Danikas DD, Karakantza M, Theodorou GL, Sakellaropoulos GC, Gogos CA.: Prognostic value of phagocytic activity of neutrophils and monocytes in sepsis. Correlation to CD64 and CD14 antigen expression. Clin Exp Immunol.154:87-97 (2008)

[25] Cid J, Garcia-Pardo G, Aguinaco R, Sanchez R.: Neutrophil CD 64: Diagnostic accuracy and prognostic value in patients presenting to the emergency department. Eur J Clin Microbiol Inf Dis; 30:845-52 (2011)

[26] Chen Q, Shi J, Fei A, Wang F, Pan S, Wang W.: Neutrophil CD64 expression is a predictor of mortality for patients in the intensive care unit. Int J Clin Exp Pathol;7(11):7806-7813 (2014)

[27] Ajmani S, Agarwal V, Gurjar M.: State of globe: Neutrophil CD64: Is it a reliable biomarker for sepsis?. J Global Infect Dis;10:33-4 (2018) 